\title{
Intrauterine growth retardation detected in several species by non-normal birthweight distributions
}

\author{
R. Wootton, P. A. Flecknell, J. P. Royston and M. John \\ M.R.C. Clinical Research Centre, Watford Rd., Harrow, Middlesex HAI 3UJ, U.K.
}

\begin{abstract}
Summary. The statistical distribution of birthweights in 64 litters of pigs, 48 litters of dogs, 59 litters of rabbits, 130 litters of rats and 46 litters of mice was examined. Birthweight followed a Gaussian or normal distribution in about two-thirds of the litters inspected, as judged visually using a normal probability plot, and by the W-test statistic. In the remainder, a single outlying observation in an otherwise normal sample was detected by Dixon's test, and/or a separate sub-population of low birthweight animals could be identified by fitting two normal populations to the observed samples. In the non-Gaussian litters, the average proportion of affected members was $40 \%$ of the litter. These results suggest that growth-retarded neonates should not be regarded simply as the tail-end of a normal distribution.
\end{abstract}

\section{Introduction}

It has been widely assumed that the birthweights of the members of a litter of a polytocous species follow a Gaussian or normal distribution. Individuals of excessively low birthweight (colloquially the 'runts' in a litter) have been assumed to represent extreme values in this distribution (Widdowson, 1974). However, investigations using mice induced to superovulate have suggested that the low birthweight animal is not simply a member of the tail-end of a continuous normal distribution of birthweights (McLaren \& Michie, 1960a), but a distinct entity. Although this might only be the consequence of the superovulation itself, analysis of the birthweight distributions of normal pigs has also shown that, in some litters, there may be a discrete sub-population of piglets of lower birthweight (Royston, Flecknell \& Wootton, 1982). This implies that growth-retarded piglets may be the consequence of an unidentified process occurring during gestation. We have extended these observations by investigating the birthweight distributions of a range of polytocous mammals.

\section{Materials and Methods}

\section{Experimental animals}

Birthweight data from 347 litters from 5 different species were examined (Table 1). All litters were weighed at full-term after natural parturition or after delivery by Caesarean section, except for 26 litters of pigs which were delivered by Caesarean section during the last 2 weeks of gestation (full-term in the pig occurs at approximately 114 days). All mothers were clinically normal, healthy animals. 
Table 1. Characteristics of the litters examined

\begin{tabular}{llcrc}
\hline & & & \multicolumn{2}{c}{ Litter size } \\
\cline { 4 - 5 } Species & \multicolumn{1}{c}{ Strain } & Litters & Mean & Range \\
\hline Pig & Large White & 64 & $11 \cdot 3$ & $5-18$ \\
Dog & Beagle & 18 & $7 \cdot 1$ & $3-10$ \\
& Irish Setter & 2 & $8 \cdot 1$ & $3-10$ \\
& Labrador & 28 & $10 \cdot 0$ & $9-11$ \\
Rabbit & New Zealand White & 59 & $7 \cdot 7$ & $3-12$ \\
Rat & Sprague-Dawley & 130 & $12 \cdot 7$ & $1-18$ \\
Mouse & BALB/c & 46 & $4 \cdot 9$ & $1-9$ \\
\hline
\end{tabular}

\section{Statistical methods}

The statistical distribution of birthweights in each litter was examined by 3 different methods. All methods were applied to all litters of sufficient size (see below).

(1) Normality. The bodyweights from litters of 3 or more young were ordered and plotted on a normal probability scale (Wilk \& Gnanadesikan, 1968). The normal plot is a graphical technique for assessing the statistical distribution of a sample. The method of construction is as follows. (1) Arrange the sample values $x_{1}, x_{2}, \ldots, x_{n}$ in increasing order of size. (2) Calculate the cumulative sample proportion, $P_{i}=100\left[\left(i-\frac{1}{3}\right) /\left(n+\frac{1}{3}\right)\right]$ for $i=1,2, \ldots, n$. (3) Either plot the ordered $x_{i}$ against the $P_{i}$ on normal probability paper, with the $P_{i}$ on the probability scale, or calculate $z_{i}=N E D\left(P_{i}\right)$, the Normal Equivalent Deviate (from standard statistical tables) of $P_{i}$, and plot the $x_{i}$ against the $z_{i}$ on ordinary linear graph paper. (4) In the context of birthweight distributions, the plot will usually be approximately straight (a single normal distribution), or 'dog-legged' on the left (two normal populations). The graphs were assessed visually and deviations from straightness were tested by the W-test of Shapiro \& Wilk (1965), a significance level being obtained by the extension of Royston (1982).

(2) Single outlier. To detect the presence of a single possible outlier in an otherwise normal distribution, Dixon's (1953) test was applied to each litter of size 3 or more. The approximate significance level of the result was obtained by interpolating the table given by Dixon (1953) using cubic spline functions (Numerical Algorithms Group, 1982).

(3) Multiple outliers. To detect multiple outliers, separate means, standard deviations and a proportion for each putative population were fitted to the observations by the method of maximum likelihood. Since 5 parameters were estimated by this method, only litters comprising at least 6 animals could be analysed. Use of the likelihood ratio test allowed the calculation of a $\chi^{2}$ statistic from which the significance level for the existence of two normal populations could be obtained. Full details are given by Royston et al. (1982).

\section{Results}

Birthweight data from 347 litters were examined. All statistical tests were applied to the 291 litters containing 6 or more fetuses, and all tests except that for detecting multiple outliers were applied to the 340 litters containing 3 or more fetuses. Data from 7 litters containing only 1 or 2 fetuses could not be analysed by any test.

Inspection of the normal plots suggested that the distribution of birthweights in the majority of litters did not deviate from normality and this was confirmed by the W-test. However, the distributions were significantly $(P<0.05)$ non-normal by one or more tests for outliers in 19 of the 64 litters of pigs, 11 of the 48 litters of dogs, 11 of the 59 litters of rabbits, 44 of the 130 litters of rats 
Table 2. Numbers of abnormal litters

\begin{tabular}{|c|c|c|c|c|c|c|c|}
\hline \multirow[b]{2}{*}{ Species } & \multicolumn{3}{|c|}{ No. of litters* } & \multicolumn{4}{|c|}{ No. of abnormal litters $\dagger$} \\
\hline & $\mathbf{n}_{3}$ & $\mathrm{n}_{6}$ & $\mathrm{n}_{\mathrm{T}}$ & $\mathrm{n}_{\mathrm{L}}$ & $\mathrm{n}_{2}$ & $\mathrm{n}_{\mathrm{H}}$ & $\mathrm{n}_{\mathrm{T}}$ \\
\hline Pig & 64 & 63 & 64 & 4 & 13 & 6 & 19 \\
\hline Dog & 48 & 43 & 48 & 5 & 7 & 0 & 11 \\
\hline Rabbit & 59 & 46 & 59 & 6 & 4 & 1 & 11 \\
\hline Rat & 127 & 122 & 130 & 23 & 16 & 5 & 44 \\
\hline Mouse & 42 & 17 & 46 & 6 & 4 & 1 & 11 \\
\hline
\end{tabular}

${ }^{*} \mathrm{n}_{3}$ is the number of litters containing 3 or more live fetuses; $\mathrm{n}_{6}$ is the number of litters containing 6 or more live fetuses; $n_{T}$ is the total number of litters.

$\dagger n_{L}$ is the number of litters containing one low birthweight outlier; $n_{2}$ is the number of the litters with two normal populations of fetuses; $n_{H}$ is the number of litters containing one high birthweight outlier; $n_{\mathrm{T}}$ is the total number of abnormal litters.

and 11 of the 46 litters of mice (Table 2). Text-figure 1 shows the mean weights of the rat litters with normally-distributed birthweights, together with the mean weights of the constituent subpopulations in litters in which non-normal distributions were detected.

\section{Discussion}

Fetal growth retardation has been reported for animals of many species including man (Gruenwald, 1963; Widdowson, 1971; Avery, 1974; Mellor \& Pearson, 1977) and is obviously important, not only to the paediatrician but also to those concerned with animal breeding and production. Growth-retarded animals have a higher mortality in the neonatal period than do their normal littermates and often fail to grow as rapidly after birth. Intrauterine growth retardation in man results in infants at greater risk from a range of metabolic disorders. In the past, growthretarded neonates have been identified either by purely subjective means (the "characteristic clinical appearance'), or by a variety of somewhat arbitrary criteria. These have included, for example, selection of those individuals whose birthweight is less than two standard deviations below the mean bodyweight for gestational age, or below the tenth percentile (Frigoletto \& Rothchild, 1977). By definition, such criteria will misidentify a substantial number of normal animals. Within litters, 'runts' have been variously defined as "the smallest in the litter" (Widdowson, 1971) or as being below a certain 'normal' birthweight (Hegarty \& Allen, 1978).

All statistical methods that have been applied so far have assumed that the distribution of birthweights is Gaussian or normal. The results of the present study confirm the finding of Royston et al. (1982) for the pig and demonstrate that sub-populations of growth-retarded animals are present in a substantial proportion of the litters of other polytocous species. In the 5 species studied, this proportion was $21,16,9,13$ and $24 \%$ for pigs, dogs, rabbits, rats and mice respectively. These values are not significantly different from each other $\left(P=0.4\right.$ using a $\chi^{2}$ test), in spite of the disparities in maternal weights and in litter sizes.

Text-figure 1 shows that the median weight of the 86 rat litters in which birthweight was distributed normally was $5.6 \mathrm{~g}$. The median weight of the 23 litters in which a single low outlier was detected by Dixon's test was $5.7 \mathrm{~g}$ (excluding the outlier), not significantly different $(P>0.05$ by the Kruskal-Wallis test). This is confirmation, therefore, that the low outlier is a fundamentally different member of the litter in which it occurs, the remainder being normal. On the other hand, there was evidence of two distinct normal sub-populations in 16 litters, the median weights of which were 5.5 and $6.1 \mathrm{~g}$. Since the median weight of the heavier is significantly greater than that for the entirely normal litters $(P<0.01)$ and the median weight of the lighter is marginally less 


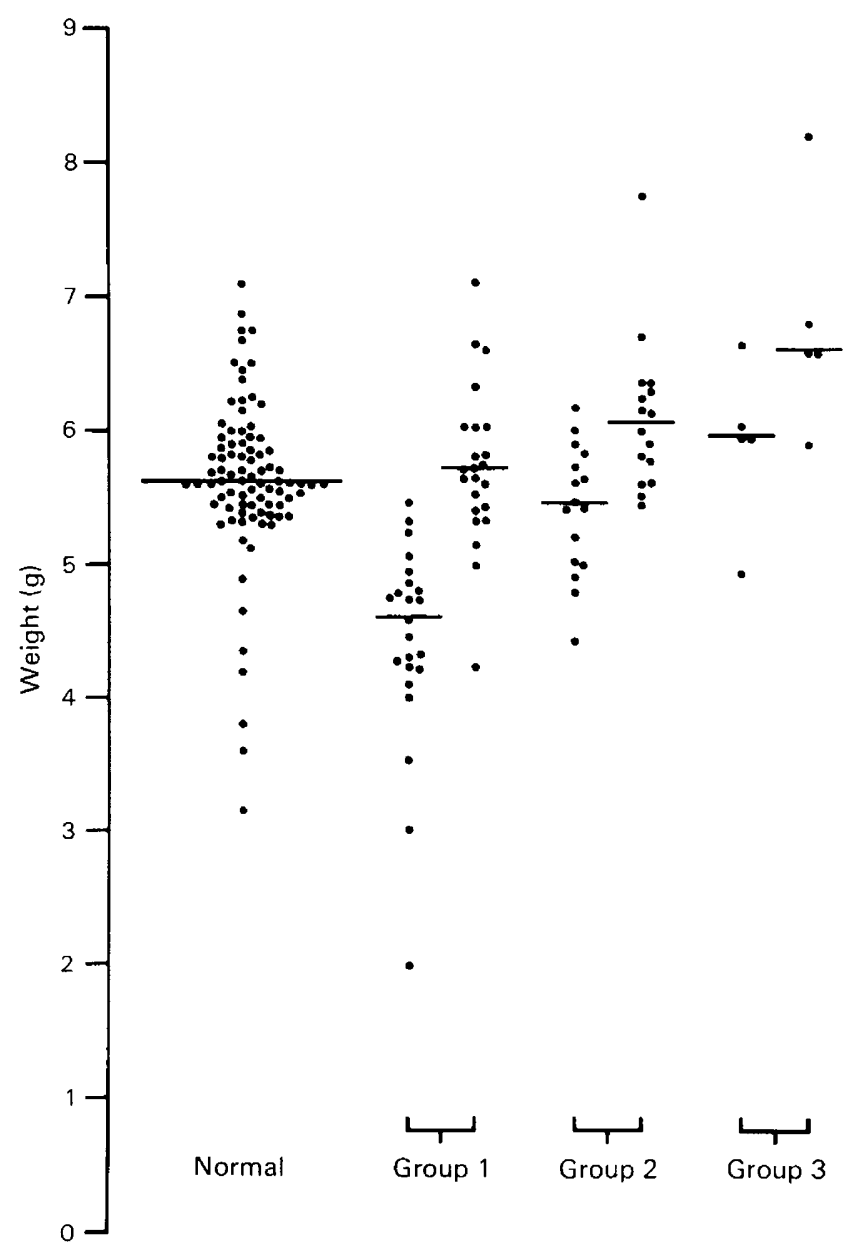

Text-fig. 1. Mean weights of the rat litters with normally-distributed birthweights, together with the mean weights of the constituent sub-populations in litters in which non-normal birthweight distributions were detected. Group 1 are those litters in which a single low outlier was detected: the left-hand column gives the weights of the low outliers while the right-hand column represents the mean weight of the remainder of the litter. Group 2 are those litters in which two normal distributions of birthweights were observed: the left-hand column gives the mean weights of the lighter distribution, the right-hand column the mean weights of the heavier. Group 3 are those litters in which a high outlier was detected: the right-hand column gives the weights of the high outlier in a given litter and the left-hand column is the mean weight of the remainder of the litter. The horizontal bars are the medians of each distribution.

$(P<0.07)$, it appears that the heavier sub-population may have grown at the expense of the lighter. The same trend was apparent in the 5 litters in which a single high outlier was detected by Dixon's test, although, because of the small numbers involved, test statistics do not have much power. While it is possible that these birthweight changes may be the result of fetal competition for finite maternal haemodynamic resources (McLaren \& Michie, 1960b; Perry \& Rowell, 1969), there is no conclusive evidence at present.

The statistical techniques we have employed enable the detection of both the extreme case of growth retardation - the so-called 'runt' of the litter, often readily distinguishable by superficial 
physical examination (Cooper, John, McFadyen \& Wootton, 1978)-as well as those animals that are only moderately affected. In 'dog-legged' normal plots which suggest two populations, it is not possible to be certain to which population a given individual belongs in the region of the 'knee'. However, the quantification of more subtle effects on the growth of the fetus may be valuable in detecting adverse influences on its development. Such analyses have almost always been based previously on average birthweight, whereas identification of less well-grown fetuses is potentially more important. The occurrence of an increased number of 'runts' was reported in an animal model of the fetal alcohol syndrome (Leichter \& Lee, 1979), although only subjective criteria were used to identify the affected animals.

Intrauterine growth retardation is a common phenomenon, occurring to a greater or lesser extent in about a quarter of all full-term litters of the polytocous species we have studied. Our findings strongly suggest that the singleton growth-retarded neonate is not a member of the same population as its littermates, but, by implication, results from an as-yet-unidentified process during gestation. Furthermore, a substantial number of litters contains more than one growth-retarded individual, and in these litters the remaining fetuses appear to be growth promoted.

We thank Dr S. E. Blaza (Animal Studies Centre, Melton Mowbray) for the dog data; the Department of Reproduction and Toxicology (Huntingdon Research Centre, Huntingdon) for the rabbit data; and Dr M. J. Halsey (Clinical Research Centre, Harrow) for the rat and mouse data.

\section{References}

Avery, M.E. (1974) Differential organ growth in littermate rabbits. In Size at Birth (Ciba Fdn Symp. No. 27), pp. 83-97. Eds K. Elliott \& J. Knight. Associated Scientific Publications, Amsterdam.

Cooper, J.E.C., John, M., McFadyen, I.R. \& Wootton, R. (1978) Early appearance of "runting" in piglets. Vet. Rec. 102, 529-530.

Dixon, W.J. (1953) Processing data for outliers. Biometrics $9,74-89$.

Frigoletto, F.D. \& Rothchild, S.B. (1977) Altered fetal growth: an overview. Clin. obstet. Gynec. 20,915-923.

Gruenwald, P. (1963) Chronic fetal distress and placental insufficiency. Biol. Neonate 5, 215-265.

Hegarty, P.V.J. \& Allen, C.E. (1978) Effect of pre-natal runting on the post-natal development of skeletal muscles in swine and rats. J. Anim. Sci. 46, 1634 1640.

Leichter, J. \& Lee, M. (1979) Effect of maternal ethanol administration on physical growth of the offspring in rats. Growth 43, 288-297.

McLaren, A. \& Michie, D. (1960a) Congenital runts. In Congenital Malformations (Ciba Fdn Symp.), pp. 178-194. Eds G. E. W. Wolstenholme \& $\mathbf{M}$. O'Connor. Churchill Livingstone, Edinburgh.

McLaren, A. \& Michie, D. (1960b) Control of pre-natal growth in mammals. Nature, Lond. 187, 363-365.

Mellor, D.J. \& Pearson, R.A. (1977) Some changes in the composition of blood during the first 24 hours after birth in normal and growth retarded lambs. Annls Rech. vét. 8, 460-467.

Numerical Algorithms Group (1982) Fortran Library Manual Mk 9. Oxford.

Perry, J.S. \& Rowell, J.G. (1969) Variations in foetal weight and vascular supply along the uterine horn of the pig. J. Reprod. Fert. 19, 527-534.

Royston, J.P. (1982) An extension of Shapiro and Wilk's W-test for Normality to large samples. Appl. Statistics 31, 115-124.

Royston, J.P., Flecknell, P.A. \& Wootton, R. (1982) New evidence that the intra-uterine growth-retarded piglet is a member of a discrete subpopulation. Biol. Neonate 42, 100-104.

Shapiro, S.S. \& Wilk, M.B. (1965) An analysis of variance test for normality (complete samples). Biometrika 52, 591-611.

Widdowson, E.M. (1971) Intra-uterine growth retardation in the pig. Biol. Neonate 19, 329-340.

Widdowson, E.M. (1974) Immediate and long-term consequences of being large or small at birth: a comparative approach. In Size at Birth (Ciba Fdn Symp. No. 27), pp. 65-82. Eds K. Elliott \& J. Knight. Associated Scientific Publications, Amsterdam.

Wilk, M.B. \& Gnanadesikan, R. (1968) Probability plotting methods for the analysis of data. Biometrika 55, $1-17$

Received 5 April 1983 